\title{
Summary of Drag Coefficients of Various Shaped Cylinders
}

\author{
C. F. Heddleson \\ D. L. Brown \\ R. T. Cliffe
}

APRIL, 1957

United States Air Force

United States Atomic Energy Commission
Contract No. AF 33(038)-21102

Confract No. AT (11-1)-171

\section{GENERAL ELECTRIC \\ ATOMIC PRODUCTS DIVISION}

AIRCRAFT NUCLEAR PROPULSION DEPARTMENT

Cincinnati 15, Ohio

Published By

Technical Publications Sub-Section

April, 1957 